Pirineos, 153-154: 123 a 143, JACA; 1999

\title{
ANÁLISIS DE LA REPELENCIA AL AGUA EN UNA PEQUEÑA CUENCA HIDROGRÁFICA AFECTADA POR FUEGO CONTROLADO EN EL ÁREA MONTAÑOSA CENTRAL DE PORTUGAL
}

\author{
A. Ceballos*, A. J. D. Ferreira**, C. O. A. Coelho** \& A. K. Boulet** \\ * Departamento de Geografía, Universidad de Salamanca. 37071-Salamanca (España) \\ ** Departamento de Ambiente e Ordenamento, Universidade de Aveiro. 3810-Aveiro (Portugal)
}

\begin{abstract}
RESUMEN.- En el presente trabajo se describe y analiza la repelencia al agua detectada en una pequeña cuenca hidrográfica afectada por fuego controlado en el área montañosa central de Portugal. También se establece una discusión acerca de los métodos utilizados para medir la hidrofobia y el grado de correspondencia entre los resultados obtenidos en el campo y en el laboratorio. Los resultados muestran la escasa influencia del fuego controlado sobre los parámetros fisicos y contenido de materia orgánica del suelo, indicando, sin embargo, una estrecha relación entre la distribución espacial de Erica sp. y suelos más hidrófobos. El grado de replicación entre la hidrofobia medida en el campo y en el laboratorio es discreto; no obstante, en el laboratorio sí se ha encontrado un buen nivel de correspondencia entre las dos técnicas utilizadas (Molarity of an Ethanol Droplet y Water Drop Penetration Time).
\end{abstract}

ABSTRACT.- In this work it is described and analyzed the water repellency observed in a small catchment subject to prescribed burning and located in mountainous central part of Portugal. As well, a discussion about the methodologies usually used to measure the soil hydrophobocity, the replicability between field and laboratory data is established. The results show the little influence in soil physical parameters and organic matter content of prescribed burning and indicate a close relationship between spatial distribution of Erica sp. and water repellency. The replicability between soil hydrophobocity data from the field and laboratory is limited; nevertheless, a high level of correspondence exists between Molarity of an Ethanol Droplet and Water Drop Penetration Time data in the laboratory.

RÉSUMÉ.- Dans cet article est décrit puis analysé le phénomène de répulsion à l'eau qui a été observé dans un petit bassin hydrographique soumis au feu contrôlé dans la zone montagneuse centrale du Portugal. Une discussion est également menée autour des méthodes utilisées pour mesurer l'hydrophobie et sur le degré de corres- 
pondance entre les résultats obtenus sur le terrain et en laboratoire. L'étude montre la faible influence du feu contrôlé sur les paramètres physiques et le taux de matière organique du sol, mais indique cependant une étroite relation entre la distribution spatiale de Erica sp. et des sols plus hydrophobes. Si le niveau de réplicabilité entre l'hydrophobie mesurée sur le terrain et en laboratoire est limité, il a cependant été mis en évidence une étroite correspondance entre les deux.techniques utilisées en laboratoire (Molarity of an Ethanol Droplet et Water Drop Penetration Time).

Key words: Water repellency; Molarity of an Ethanol Droplet, Water Drop Penetration Time, prescribed burning, Erica sp., small catchment, Portugal.

\section{Introducción}

Aunque no es muy frecuente, la repelencia al agua o hidrofobia es una propiedad del suelo que puede tener notables consecuencias hidrológicas, geomorfológicas, ecológicas y económicas según su grado de intensidad.

Como indica DOERR (1997), existen algunos trabajos aislados en la primera mitad del presente siglo que detectan comportamientos hidrófobos asociados a determinados usos del suelo, pero no es hasta la Conferencia de Riverside (California-USA), a finales de los 60 , cuando comienzan a proliferar numerosos estudios sobre el tema, especialmente en Estados Unidos y Australia (de BANO \& LETEY, 1969).

La aparición de la hidrofobia está asociada a la precipitación de sustancias orgánicas en el suelo a través de varios mecanismos: i) la materia orgánica particulada procedente de las plantas se adhiere a las partículas minerales del suelo formándose una película hidrófoba (FRANCO et al., 1994); ii) la colonización de ciertas partes del suelo por determinados tipos de hongos (WILLKINSON \& MILLER, 1978; YORK, 1998) y iii) el desarrollo de incendios forestales y fuegos controlados puede provocar la aparición de hidrofobia en suelos previamente hidrófilos o bien atenuar o destruir las capas hidrófobas del suelo tras el desarrollo del incendio, ya que la influencia del fuego sobre la hidrofobia está estrechamente relacionada con la temperatura alcanzada durante la combustión (de BANO et al., 1976).

La extensión geográfica de la hidrofobia es amplia, siendo especialmente significativa en zonas áridas y semiáridas, destacando los casos de Estados Unidos (p. ej. de BANO et al., 1970), Australia (p. ej. CROCKFORD et al., 1991; MOORE \& BLACKWELL, 1998) y África del Sur (p. ej. SCOTT, 1991 y 1993). No obstante, en medios húmedos y súbhúmedos también ha sido detectada (p. ej. JUNGERIUS \& DEJONG, 1989; DEKKER, 1998; RITSEMA, 1998). En el 
ANÁLISIS DE LA REPELENCIA AL AGUA EN UNA PEQUEÑA CUENCA HIDROGRÁFICA..

caso de la Península Ibérica destacan los estudios desarrollados en el noreste de España en bosques mediterráneos de Quercus suber (SEVINK et al. 1989; IMESON et al., 1992), en el suroeste de España en dehesas de Quercus rotundifolia (CERDÀ et al. 1998) y en la región centro de Portugal asociados a Eucalyptus globulus y Pinus pinaster (DOERR et al., 1996 y 1998; FERREIRA, 1996; FERREIRA et al., 1998).

Una de las características fundamentales de la hidrofobia es su discontinuidad temporal y espacial. Temporalmente, está asociada a períodos secos que permitan la formación de una capa hidrófoba mínimamente estable, ya que como demuestran WALSH et al. (1994) durante lluvias continuadas y altos contenidos de agua en el suelo la hidrofobia desaparece, para reaparecer una vez que las lluvias cesan y los suelos se secan (CROCKFORD et al., 1991).

Aparte de la existencia de sustancias hidrófobas, el suelo tiene que ser susceptible a la formación de una película repelente al agua (WALLIS \& HORNE, 1992): suelos de texturas groseras (contenido de arcilla inferior al $5 \%$ ) favorecen la adhesión de las sustancias hidrófobas a las partículas minerales ( MOORE \& BLACKWELL, 1998).

Las implicaciones hidrológicas de la hidrofobia son complejas, dependiendo del grado de intensidad. De modo general reduce la capacidad de infiltración de un suelo, aumentando la producción de escorrentía superficial y los riesgos erosivos (BURCH et al., 1989; IMESON et al., 1992; MEEUWING, 1971; SCOTT et al., 1998, SHAKESBY et al., 1993). A escala puntual favorece lós flujos a través de macroporos dando lugar a frentes de humedad heterogéneos (HENDRICX et al., 1993), aunque los suelos no se encuentren saturados (WILSON et al., 1990). En un nivel ecológico y también econónomico ello puede repercutir en la pérdida de solutos en superficie que alcanzan rápidamente el nivel freático con riesgos de contaminación de las aguas subterráneas. La variabilidad espacial de la hidrofobia, con partes del terreno secas y partes húmedas, puede implicar una pobre germinación de cultivos y pastos. Finalmente destacan sus implicaciones geomorfológicas, ya que se han detectado la formación de regueros y cárcavas en determinados tipos de suelo que de no ser fuertemente hidrófobos no hubieran tenido este tipo de modelado (MOORE \& BLACKWELL, 1998).

El objetivo principal de este trabajo es la descripción y análisis de la repelencia al agua detectada en las vertientes de una pequeña cuenca hidrográfica situada en el área montañosa central de Portugal. Se estudia la intensidad del fenómeno, su variabilidad temporal y espacial, y se discute acerca de sus principales implicaciones hidrogeomorfológicas. También se establece una discusión acerca de los métodos y técnicas habitualmente utilizados para detectar la hidrofobia y de la correspondencia entre los resultados obtenidos 
in situ - sobre el propio terreno- y en el laboratorio tras el análisis de una serie de muestras.

La investigación se encuadra en un proyecto más amplio que está estudiando las repercusiones hidrológicas y erosivas del fuego controlado y la posibilidad de reintroducir el pastoreo con cabras en tierras marginales de montaña (COELHO et al., 1998).

\section{Zona de estudio}

La cuenca experimental de Valtorto se encuentra localizada en la ladera norte de la Sierra de Lousâ, en la zona central de Portugal (Figura 1 y Foto 1).

Con un tamaño de 6,8 hectáreas, la cuenca se sitúa entre los 600 y $730 \mathrm{~m}$ de altitud. El sustrato geológico lo constituye el Complejo Esquisto Grauváquico y los suelos se caracterizan por ser poco profundos $(0-30 \mathrm{~cm})$ y pedregosos, con afloramientos parciales de roca, clasificándose como litosuelos. Las pendientes de las laderas son acentuadas, oscilando en torno a los $20^{\circ}$.

La precipitación anual media es de $1161 \mathrm{~mm}$, con una importante concentración en los meses invernales (40,6\%). La estación seca se reduce a los meses de julio y agosto. La temperatura anual media oscila en torno a los $13,5^{\circ} \mathrm{C}$.

La mayor parte de la cuenca $(88 \%$ ) fue quemada de forma controlada en abril de 1996, siendo actualmente pastoreada de forma esporádica por peque-

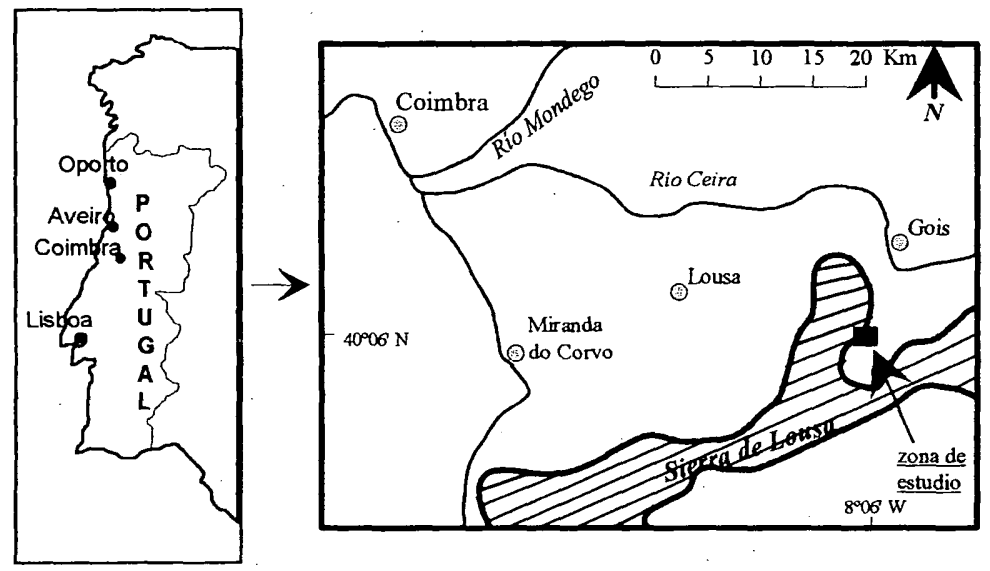

Figura 1. Mapa de localización de la zona de estudio. Location map of study site. 


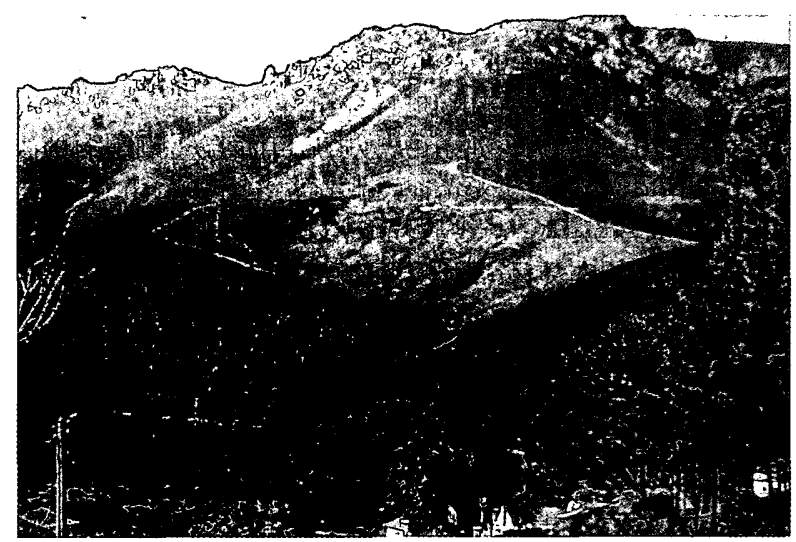

Foto 1. Vista general de la cuenca experimental de Valtorto. General view of Valtorto experimental catchment.

ños rebaños de cabras de las aldeas cercanas. En esta unidad predomina el suelo desnudo sobre el cubierto y aparecen de forma dispersa manchas de Chamaespartium tridentatum, Erica umbellata, Erica cinerea, Erica lusitanica, Genista triacanthos y diversas especies de gramíneas. La parte restante de la cuenca está ocupada por un brezal muy denso, constituido por las especies anteriores, en proceso avanzado de regeneración tras un incendio forestal sufrido en 1990.

\section{Métodos y técnicas}

Entre los diversos métodos destinados a detectar y medir el grado de hidrofobia de un suelo, los dos más extendidos por la posibilidad de ser aplicados directamente en el campo y fácil ejecución son Water Drop Penetration Time (WDPT) y Molarity of an Ethanol Droplet (MED). El resto de los métodos existentes son de laboratorio y su aplicación requiere de un protocolo más complicado.

El WDPT test consiste en medir el tiempo que invierte en infiltrarse completamente una gota de $0,05 \mathrm{ml}$ de agua destilada colocada sobre la superficie del suelo (LETEY, 1969). Si la gota se infiltra en un margen de tiempo corto (menos de 5 segundos) nos encontraremos ante un suelo muy hidrófilo. Si demora más tiempo se podrá estimar el grado de persistencia o estabilidad de la hidrofobia, utilizando una serie de clases cuyos rangos varían sensiblemente según autores (Tabla 1). Este hecho constituye un serio inconveniente 
PIRINEOS 153-154

\begin{tabular}{|c|c|c|c|c|c|c|c|}
\hline \multicolumn{2}{|c|}{ Adams et al. (1969) } & \multicolumn{2}{|c|}{ Roberts \& Carbon (1971) } & \multicolumn{2}{|c|}{ Barret E Slaymaker (1989) } & \multicolumn{2}{|c|}{ Bisdom et al. (1993) } \\
\hline (segundos) & Clasificación & (segundos) & Clasificación & (segundos) & Clasificación & (segundos) & Clasificación \\
\hline$<10$ & Hidrófilo & $<1$ & Hidrófilo & $<10$ & Hidrófilo & $<5$ & Hidrófilo \\
\hline $10-60$ & $\begin{array}{l}\text { Ligeramente } \\
\text { hidrófobo }\end{array}$ & $1-10$ & $\begin{array}{l}\text { Ligeramente } \\
\text { hidrófobo }\end{array}$ & $10-600$ & $\begin{array}{l}\text { Hidrofobia } \\
\text { transitoria }\end{array}$ & $5-60$ & $\begin{array}{l}\text { Ligeramente } \\
\text { hidrófobo }\end{array}$ \\
\hline \multirow[t]{3}{*}{$>60$} & $\begin{array}{l}\text { Fuertemente } \\
\text { hidrófobo }\end{array}$ & $11-60$ & $\begin{array}{c}\text { Fuertemente } \\
\text { hidrófobo }\end{array}$ & $>600$ & Hidrófobo & $60-600$ & $\begin{array}{c}\text { Fuertemente } \\
\text { hidrófobo }\end{array}$ \\
\hline & & $>60$ & $\begin{array}{l}\text { Severamente } \\
\text { hidrófobo }\end{array}$ & : & & $600-3600$ & $\begin{array}{c}\text { Severamente } \\
\text { hidrófobo }\end{array}$ \\
\hline & & & & & & $>3600$ & $\begin{array}{c}\text { Extremadamente } \\
\text { hidrofobo }\end{array}$ \\
\hline
\end{tabular}

Tabla 1. Clasificaciones de diferentes autores para medir la hidrofobia aplicando el WDPT test. Classifications by different authors of water repellency on the basis of WDPT test.

a la hora de comparar los resultados obtenidos con los aportados por otras fuentes. En este trabajo utilizaremos una de las clasificaciones propuestas en DOERR (1998) por su nivel de detalle y la posibilidad de ser comparada con los resultados obtenidos mediante el MED test (Tabla 2).

El MED test (KING, 1981) detecta principalmente el grado de intensidad de la repelencia. Para ello se necesita una serie de frascos con diversas concentraciones de alcohol etílico mezclado con agua destilada (por ejemplo 0,3 , 5,8 '5 13,24 y $36 \%$ de volumen). Sobre la superficie de un suelo hipotéticamente hidrófobo se van colocando sucesivamente gotas con concentraciones crecientes de alcohol hasta que la gota tarde menos de 3 segundos en infiltrarse. La técnica se basa en que el alcohol etílico disminuye la tensión superficial de la gota y se puede establecer una escala de hidrofobia constituida por 7 clases correspondientes a las 7 concentraciones utilizadas (Tabla 3).

Para detectar, medir y analizar la repelencia al agua en la zona estudiada se ha seguido la siguiente sistemática:

\begin{tabular}{|lccccccc|}
\hline Clasificación & $\begin{array}{c}\text { Muy } \\
\text { hidrófilo }\end{array}$ & Hidrófilo & $\begin{array}{c}\text { Ligeramente } \\
\text { hidrófobo }\end{array}$ & $\begin{array}{c}\text { Moderadamente } \\
\text { hidrófobo }\end{array}$ & Muy hidrófobo & $\begin{array}{c}\text { Fuertemente } \\
\text { hidrófobo }\end{array}$ & $\begin{array}{c}\text { Extremadamente } \\
\text { hidrófobo }\end{array}$ \\
\hline $\begin{array}{l}\text { Clases } \\
\text { Intervalos }\end{array}$ & 1 & 2 & 3 & 4 & 5 & 6 & 7 \\
(segundos) & $<5$ & $5-60$ & $60-180$ & $180-600$ & $600-3600$ & $3600-18000$ & $>18000$ \\
\hline
\end{tabular}

Tabla 2. Umbrales de tiempo de infiltración y determinación de clases para el estudio de la hidrofobia mediante el WDPT test. Clasificación basada en DOERR (1998).

Threshold infiltration times and establishment of repellency classes to study water repellency using WDPT. Classification based after DOERR (1998). 
ANÁLISIS DE LA REPELENCIA AL AGUA EN UNA PEQUEÑA CUENCA HIDROGRÁFICA...

\begin{tabular}{|ccl|}
\hline Concentración de alcohol (\%) & Clase & Clasificación \\
\hline 0 & 1 & Muy.hidrófilo \\
3 & 2 & Hidrófilo \\
5 & 3 & Ligeramente hidrófobo \\
8,5 & 4 & Moderadamente hidrófobo \\
13 & 5 & Muy hidrófobo \\
24 & 6 & Fuertemente hidrófobo \\
36 & 7 & Extremadamente hidrófobo \\
\hline
\end{tabular}

Tabla 3. Clasificación de la hidrofobia según el MED test. Hydrophobicity classification based on the MED test.

En el campo se han realizado series de 20 mediciones (10 en la zona de fuego controlado y 10 en la zona regenerada) utilizando el MED test en diversos momentos del año y con diferentes niveles de humedad del suelo. Se seleccionaba aleatoriamente una superficie de $10 \mathrm{~cm}^{2}$, limpiando cuidadosamente con la mano los restos de materia orgánica y piedras, con el objetivo de exponer el suelo desnudo, para aplicar gotas con diversas concentraciones de alcohol. Posteriormente se realizaban en cada punto 3 mediciones de la humedad volumétrica del suelo por medio de un TDR (Time Domain Reflectometry), modelo Theta Probe con una sonda de 4 varillas y $5,9 \mathrm{~cm}$ de longitud, y se recogía una muestra de suelo con un anillo de $5 \mathrm{~cm}$ de profundidad y $80 \mathrm{~cm}^{3}$ de volumen para determinar en el laboratorio la densidad-porosidad, porcentaje de materia orgánica y la fracción de material mayor de $2 \mathrm{~mm}$.

Con el objetivo de analizar la variabilidad espacial de la hidrofobia, en los meses de julio y agosto de 1998, tras un período prolongado sin lluvias, se realizaron dos transectos de $100 \mathrm{~m}$ de longitud siguiendo la dirección de la vertiente (uno en la zona de fuego controlado y otro en la zona de matorral regenerado). Cada 5 metros se midió la hidrofobia y humedad del suelo, según la descripción anterior, y se tomaron dos muestras de suelo para analizar en el laboratorio, junto a las determinaciones anteriores, la distribución de arenas, limos y arcillas y estudiar la reciprocidad o no del MED y WDPT tests. También se realizaron en tramos de $25 \mathrm{~m}$ diversos muestreos de vegetación para estimar el porcentaje de cobertura del suelo por medio de una malla metálica y medir la altura de las plantas.

En el laboratorio las muestras han sido secadas al aire durante 48 horas. No es conveniente el uso de estufas ya que altas temperaturas pueden afectar a las sustancias hidrófobas (DEKKER et al., 1998). Con un tamiz de $2 \mathrm{~mm}$ de calibre se han apartado las fracciones fina y gruesa. Se han colocado 10 gr de 
material fino sobre una caja Petri de $50 \mathrm{~mm}$ de diámetro y $10 \mathrm{~mm}$ de profundidad, alisándose la superficie cuidadosamente con la mano. En un lado de la muestra se ha determinado la severidad de la hidrofobia por medio del MED test y en otro lado se han aplicado 5 gotas de agua destilada cronometrándose su tiempo de infiltración y hallándose la media para cada muestra.

Este diseño experimental permite apreciar el grado de reciprocidad de los resultados de ambos tests y el estudio de la variabilidad espacial de la hidrofobia en tres escalas: i) entre usos del suelo, ii) a escala métrica, entre los puntos de cada transecto, y iii) a escala centimétrica, en las muestras analizadas en el laboratorio.

\section{Resultados}

\subsection{Descripción de los parámetros edáficos y cobertura del suelo}

La tabla 4 muestra un gran paralelismo en todos los parámetros edáficos medidos en las dos unidades espaciales consideradas a pesar de la importante diferencia observada en la cobertura vegetal tras el fuego controlado en gran parte de la cuenca (Tabla 5).

Tras 40 días sin precipitaciones apreciables en la cuenca, los valores de humedad medidos durante los meses de julio y agosto pueden equipararse al

\begin{tabular}{|c|c|c|c|c|c|c|c|c|}
\hline \multicolumn{9}{|c|}{ Zona de Fuego Controlado } \\
\hline & $\begin{array}{l}\text { Humedad } \\
(\%)\end{array}$ & $\begin{array}{c}\text { Densidad } \\
\mathrm{g} \mathrm{cm}^{-3}\end{array}$ & $\begin{array}{c}\text { Pororidnd } \\
(\%)\end{array}$ & $\begin{array}{l}\text { M.Org } \\
(\%)\end{array}$ & $\begin{array}{c}>2 \mathrm{~mm} \\
(\%)\end{array}$ & $\begin{array}{c}\text { Arena } \\
(\%)\end{array}$ & $\begin{array}{l}\text { Limo } \\
(\%)\end{array}$ & $\begin{array}{c}\text { Arcilla } \\
(\%)\end{array}$ \\
\hline media $(n=20)$ & 3,0 & 0,89 & 65,6 & 12,1 & 56,0 & 46,6 & 41,4 & 12,0 \\
\hline STD & 2,0 & 0,16 & 6,2 & 2,4 & 15,5 & 4,9 & 5,7 & 2,8 \\
\hline C.V. $(\%)$ & 66,5 & 18,1 & 9,5 & 20,2 & 27,7 & 10,5 & 13,8 & 23,5 \\
\hline \multicolumn{9}{|c|}{ Zona Regenerada } \\
\hline & $\begin{array}{l}\text { Humednd } \\
(\%)\end{array}$ & $\begin{array}{c}\text { Densidad } \\
\mathrm{g} \mathrm{cm}^{-3}\end{array}$ & $\begin{array}{l}\text { Pororidnd } \\
(\%)\end{array}$ & $\begin{array}{l}\text { M.Org } \\
(\%)\end{array}$ & $\begin{array}{c}>2 \mathrm{~mm} \\
(\%)\end{array}$ & $\begin{array}{c}\text { Arena } \\
(\%)\end{array}$ & $\begin{array}{l}\text { Limo } \\
(\%)\end{array}$ & $\begin{array}{c}\text { Arcilla } \\
(\%)\end{array}$ \\
\hline media $(n=20)$ & 4,8 & 0,93 & 64,1 & 13,1 & 55,2 & 50,5 & 39,4 & 10,1 \\
\hline STD & 2,4 & 0,15 & 5,7 & 3,6 & 11,8 & 6,2 & 5,3 & 1,8 \\
\hline C.V. (\%) & 49,7 & 15,9 & 8,9 & 27,1 & 21,4 & 12,3 & 13,4 & 17,7 \\
\hline
\end{tabular}

Tabla 4. Comparación de las propiedades físicas del suelo y contenido de materia orgánica entre la zona sometida a fuego controlado y la zona regenerada. Comparison of soil physical properties and organic matter content between prescribed burning site and regrowth site. 
ANÁLISIS DE LA REPELENCIA AL AGUA EN UNA PEQUEÑA CUENCA HIDROGRÁFICA...

agua higroscópica del suelo, siendo estos ligeramente superiores en la zona regenerada. Los suelos en ambas unidades son poco densos, con una porosidad media-alta, valores sensiblemente altos de materia orgánica y un porcentaje de fracción gruesa $(>2 \mathrm{~mm}$ ) considerable, siendo relativamente frecuente la existencia de gravas con una longitud de $40 \mathrm{~mm}$. La distribución textural de la materia fina también es similar, clasificándose el suelo de ambas zonas como franco.

En la tabla 5 se aprecia el predominio superficial del suelo desnudo $(58,1$ $\%$ ) en el transecto de fuego controlado, siendo Chamaespartium tridentatum la especie arbustiva predominante, tanto en cobertura superficial como en porte. La situación cambia radicalmente en el área regenerada, ya que la superficie media de suelo desnudo es de sólo un 6,4 \%, dominando claramente Erica sp. en el cortejo florístico. En los tramos inferiores de ambos perfiles se observa un aumento en la cobertura vegetal y en el porte de las plantas debido a la mayor profundidad del suelo.

\begin{tabular}{|c|c|c|c|c|c|c|c|c|c|c|}
\hline \multicolumn{11}{|c|}{ Zona de Fuego Controlado } \\
\hline & \multicolumn{5}{|c|}{ Cobertura del Suelo (\%) } & \multicolumn{5}{|c|}{ Altura de las Plantas $(\mathrm{cm})$} \\
\hline & $\overline{0-25}$ & $25-50$ & $50-75$ & $75-100$ & $\overline{\text { media }}$ & $\overline{0-25}$ & $25-50$ & $50-75$ & $75-100$ & $\overline{\text { media }}$ \\
\hline Suelo Desnudo & 60,4 & 62,2 & 54,8 & 54,8 & 58,1 & 0,0 & 0,0 & 0,0 & 0,0 & 0,0 \\
\hline Erica sp. & 9,4 & 8,8 & 10,8 & 11,0 & 10,0 & 10,0 & 7,9 & 17,0 & 17,0 & 13,0 \\
\hline Ch. tridentatum & 23,2 & 20,6 & 28,4 & 23,0 & 23,8 & 14,2 & 15,1 & 27,7 & 19,0 & 19,0 \\
\hline Herbáceas & 7,0 & 8,4 & 1,8 & 4,4 & 5,4 & 7,5 & 8,1 & 6,5 & 9,3 & 7,9 \\
\hline P. pinaster & 0,0 & 0,0 & 0,0 & 0,0 & 0,0 & 0,0 & 0,0 & 0,0 & 0,0 & 0,0 \\
\hline G. triacanthos & 0,0 & 0,0 & 4,2 & 6,8 & 2,8 & 0,0 & 0,0 & 25,5 & 17,6 & 10,8 \\
\hline \multicolumn{11}{|c|}{ Zona Regenerada } \\
\hline & \multicolumn{5}{|c|}{ Cobertura del Suelo (\%) } & \multicolumn{5}{|c|}{ Altura de las Plantas $(\mathrm{cm})$} \\
\hline & $0-25$ & $25-50$ & $50-75$ & $75-100$ & media & $0-25$ & $25-50$ & $50-75$ & $75-100$ & media \\
\hline Suelo Desnudo & 4,0 & 4,2 & 6,4 & 10,8 & 6,4 & 0,0 & 0,0 & 0,0 & 0,0 & 0,0 \\
\hline Erica sp. & 66,2 & 65,2 & 58,0 & 77,0 & 66,6 & 28,2 & 40,0 & 45,3 & 45,5 & 39,7 \\
\hline Ch. tridentatum & 26,6 & 29,4 & 29,6 & 11,4 & 24,3 & 30,0 & 37,5 & 55,4 & 53,3 & 44,1 \\
\hline Herbáceas & 0,0 & 0,0 & 0,0 & 0,0 & 0,0 & 0,0 & 0,0 & 0,0 & 0,0 & 0,0 \\
\hline P. pinaster & 1,8 & 0,0 & 2,8 & 0,0 & 1,2 & 60,0 & 0,0 & 130,0 & 0,0 & 47,5 \\
\hline G. triacanthos & 1,4 & 1,2 & 3,2 & 0,8 & 1,7 & 43,2 & 42,5 & 53,3 & 0,0 & 34,8 \\
\hline
\end{tabular}

Tabla 5. Comparación de la cobertura del suelo (\%) y la altura de las plantas (cm) entre la zona sometida a fuego controlado y la zona regenerada.

Comparison of ground cover $(\%)$ and plant height $(\mathrm{cm})$ between prescribed burning site and regrowth site. 


\subsection{Características hidrófobas de los suelos}

Los gráficos 2 y 3 reflejan una notable diferencia espacial de la distribución de la hidrofobia in situ en los suelos de la cuenca. Si en la zona regenerada, con predominio de Erica sp., el $75 \%$ de las muestras tiene diverso grado de hidrofobia (clases 3,4 y 5), en el caso de la zona de fuego controlado sólo el $10 \%$ fue ligeramente hidrófobo (clase 3 ).

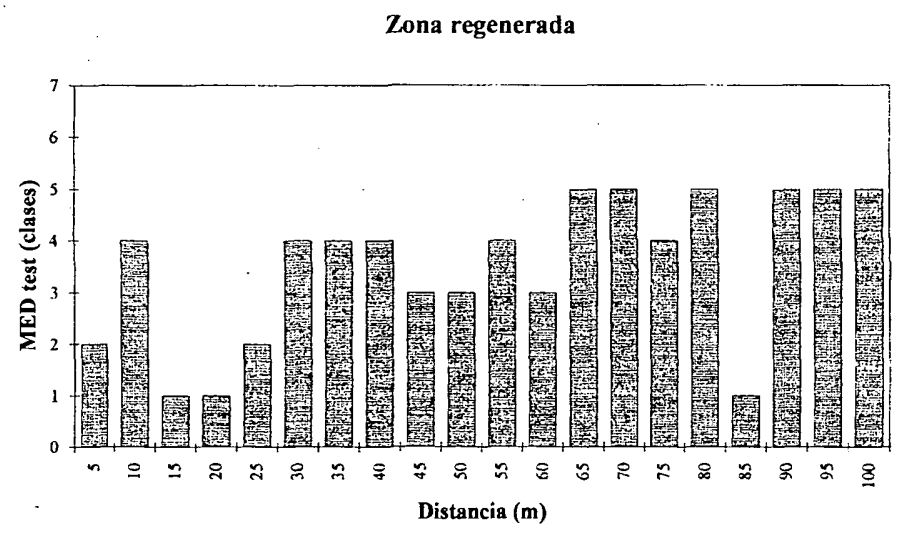

Figura 2. Distribución de la hidrofobia a lo largo del transecto en la superficie del suelo en la zona regenerada. Spatial distribution of surface soil hydrophobocity along the transect in the regrowth site.

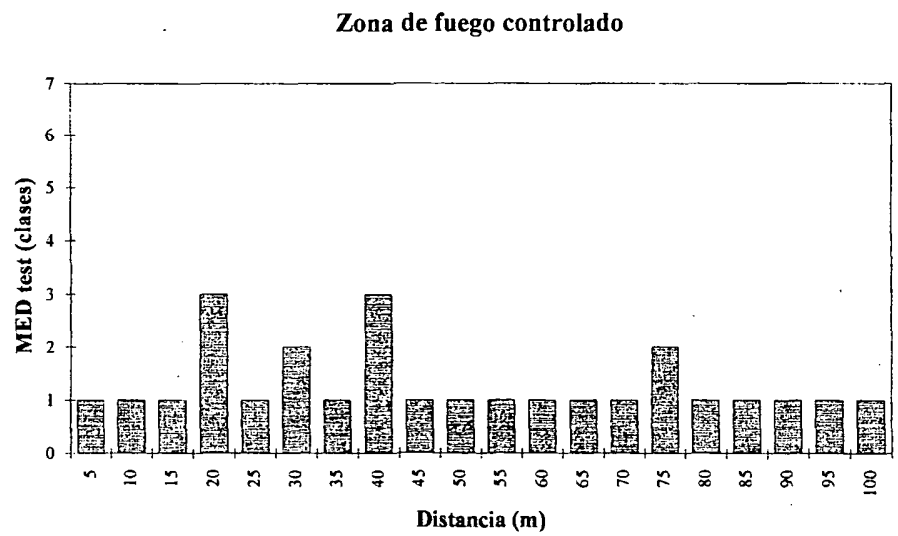

Figura 3. Distribución de la hidrofobia a lo largo del transecto en la superficie del suelo en la zona sometida a fuego controlado.

Spatial distribution of surface soil hydrophobocity along the transect in the prescribed burning site. 
En la zona regenerada se detecta una tendencia creciente de la hidrofobia conforme avanza el transecto, ya que todos los puntos "muy hidrófobos" (clase 5) se localizan en la mitad inferior del mismo (entre los 65 y $100 \mathrm{~m}$ ). En la tabla 5 se aprecia como en la primera mitad del transecto, correspondiente a la zona superior de la ladera, los ejemplares de Erica sp. tienen una talla menor que los situados en la mitad inferior.

En la zona sometida a fuego controlado no podemos establecer ninguna asociación entre hidrofobia y presencia de arbustos (Chamaespartium tridentatum). Las mediciones realizadas en los 10, 20, 30, 35, 50, 60, 70 y $100 \mathrm{~m}$ del transecto se caracterizan por ofrecer una superficie de muestreo dentro del área de proyección de la mata, y sin embargo no se detectó un comportamiento diferencial atribuible a ese factor.

En la determinación de los diversos factores que explican la aparición e intensidad de la hidrofobia se han obtenido los siguientes resultados. En los suelos de Valtorto, con una porosidad en torno al $65 \%$, la hidrofobia se manifiesta con niveles de humedad inferiores al $14 \%$ (Figura 4), aunque una vez que se baja de ese nivel no se aprecia ningún tipo de relación estadística entre hidrofobia y humedad del suelo, ya que en el caso del área regenerada (donde la hidrofobia aparece con claridad) observamos puntos calificados como "muy hidrófobos" en un rango de humedad comprendido entre 12,8 y $1 \%$.

No se ha detectado ningún tipo de relación entre hidrofobia y cantidad de materia orgánica en las muestras correspondientes a los transectos realizados (Figura 5), apreciándose altos valores de hidrofobia (clase 5) en puntos muy próximos tanto a los valores máximos como mínimos $(18,5$ y 7,6 \% respectivamente).

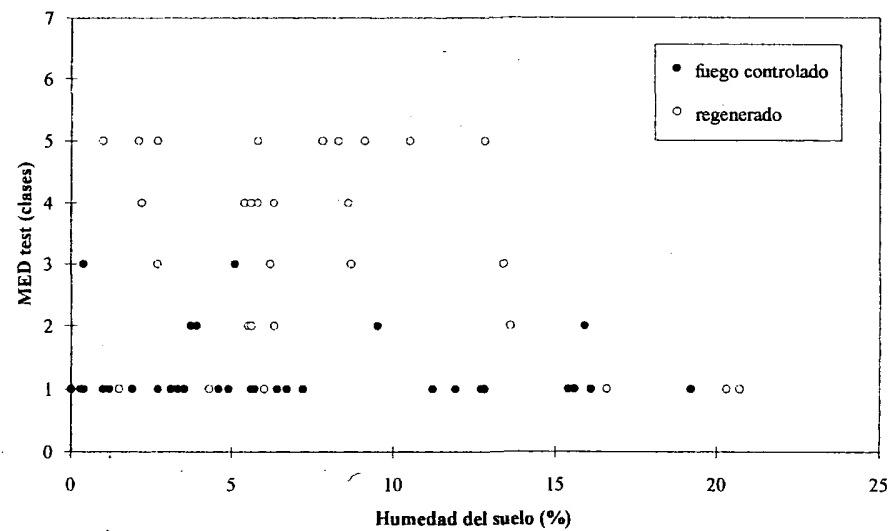

Figura 4. Relación entre humedad volumétrica del suelo y la hidrofobia (Molarity of an Ethanol Droplet) en ambos usos del suelo. Relation between volumetric soil moisture and water repellency (Molarity of an Ethanol Droplet) in both landuses. 
Otro de los factores que influye en la aparición de la hidrofobia es la distribución textural del suelo. Generalmente se asocia la aparición de la hidrofobia a suelos con un contenido de arcillas inferior al $5 \%$ debido a que las partículas arenosas favorecen la adhesión de sustancias hidrófobas. En la Figura 6 se comprueba cómo el total de las muestras analizadas supera el $5 \%$ en arcillas y no existe ninguna relación entre la fracción textural y los valores más altos de hidrofobia.

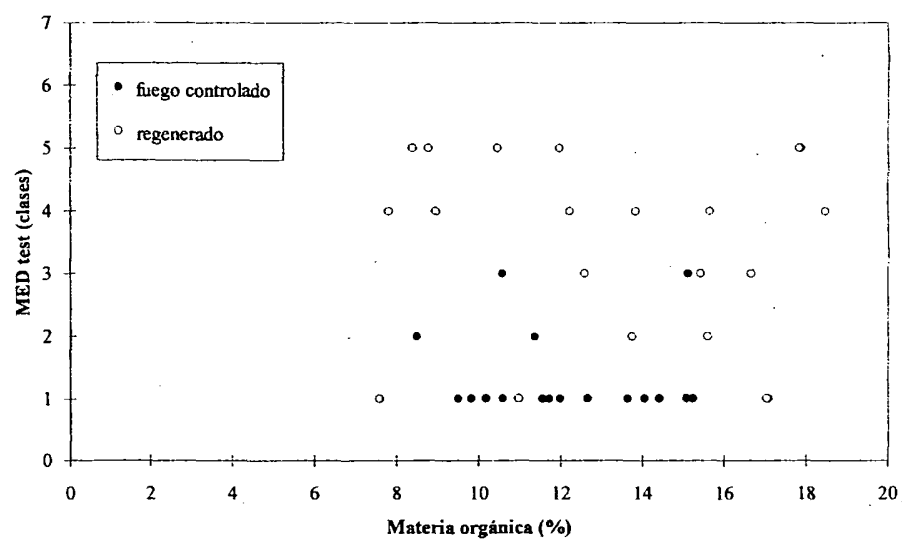

Figura 5. Relación entre contenido de materia orgánica del suelo y la hidrofobia (Molarity of an Ethanol Droplet) en ambos usos del suelo.

Relation between organic matter content in the soil and water repellency (Molarity of an Ethanol Droplet) in both landuses.

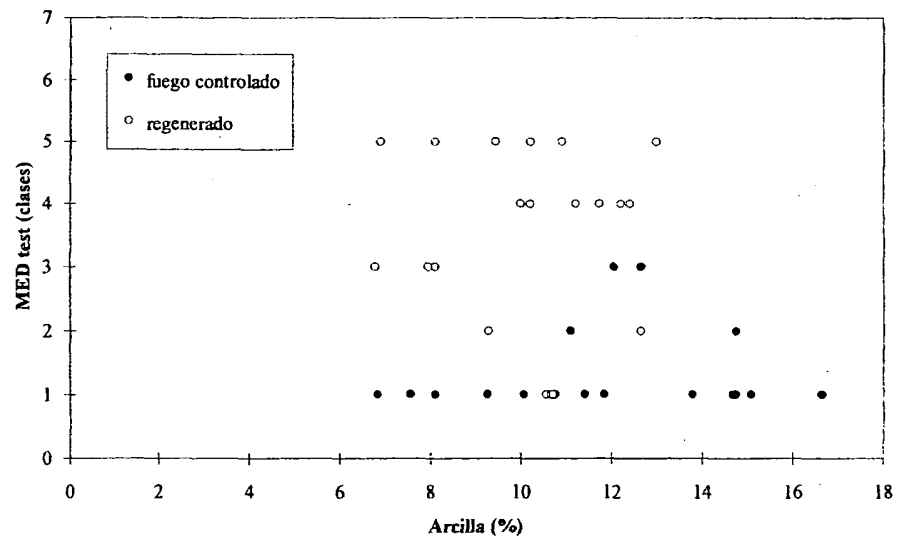

Figura 6. Relación entre contenido de arcilla en el suelo y la hidrofobia (Molarity of an Ethanol Droplet) en ambos usos del suelo.

Relation between clay content in the soil and water repellency (Molarity of an Ethanol Droplet) in both landuses. 
4.3 Comparación entre métodos de medición y grado de replicación entre los resultados obtenidos en el campo (in situ) y en el laboratorio

La comparación del nivel de hidrofobia medido en el campo y en el laboratorio con el MED test muestra un grado de replicación del 62,5\% considerando el margen de una clase (Tabla 6).

$\mathrm{Al}$ respecto, la Tabla 7 muestra que si en la zona regenerada el nivel de hidrofobia detectado es parecido, tanto en el campo como en el laboratorio, no ocurre lo mismo en la zona sometida a fuego controlado, ya que en el laboratorio se obtienen unos niveles de hidrofobia notablemente mayores: el $90 \%$ de las muestras medidas en el campo son hidrófilas (clases 1 y 2), mientras que en el laboratorio la cifra se reduce al $55 \%$.

\begin{tabular}{|lcc|}
\hline Comparación & $\begin{array}{c}\text { Número de individuos } \\
(N=80)\end{array}$ & $\begin{array}{c}\text { Distribución } \\
(\%)\end{array}$ \\
\hline clases similares & 14 & 17,5 \\
lab. < in situ (1 clase) & 8 & 10,0 \\
lab. > in situ (1 clase) & 28 & 35,0 \\
lab. < in situ (2 clases) & 4 & 5,0 \\
lab. > in situ (2 clases) & 10 & 12,5 \\
lab. < in situ (3 clases) & 2 & 2,5 \\
lab. > in situ (3 clases) & 6 & 7,5 \\
lab. < in situ (4 clases) & 4 & 5,0 \\
lab. > in situ (4 clases) & 4 & 5,0 \\
\hline
\end{tabular}

Tabla 6. Comparación de los datos obtenidos mediante el MED test tanto en el campo como en el laboratorio.

Comparison of the field and laboratory MED test results.

\begin{tabular}{|c|c|c|c|c|}
\hline \multirow[b]{2}{*}{ Clases } & \multicolumn{2}{|c|}{ Zona regenerada $(N=40)$} & \multicolumn{2}{|c|}{ Zona fuego controlado $(N=40)$} \\
\hline & In situ & Laboratorio & In situ & Laboratorio \\
\hline 1 & 6 & 6 & 32 & 12 \\
\hline 2 & 4 & 6 & 4 & 10 \\
\hline 3 & 6 & 10 & 4 & 6 \\
\hline 4 & 12 & 12 & 0 & 8 \\
\hline 5 & 12 & 6 & 0 & 4 \\
\hline 6 & 0 & 0 & 0 & 0 \\
\hline 7 & 0 & 0 & 0 & 0 \\
\hline
\end{tabular}

Tabla 7. Distribución espacial de la hidrofobia en las zonas estudiadas (datos de campo y laboratorio).

Spatial distribution of water repellency at the two study sites (field and laboratory data). 
La comparación de los resultados obtenidos en el laboratorio aplicando los MED y WDPT tests demuestra un ajuste alto entre ambas mediciones como puede apreciarse en la Figura 7, con una variación que nunca supera el margen de una clase.

Finalmente, la variabilidad de la hidrofobia en el laboratorio mediante la aplicación en una misma muestra preparada de 5 gotas de agua destilada es muy baja, debido a que en el $74 \%$ de los casos todas las gotas se agruparon en una sola de las clases descritas en la Tabla 2 y en el $100 \%$ de los casos las 5 gotas se agruparon en dos clases contiguas.

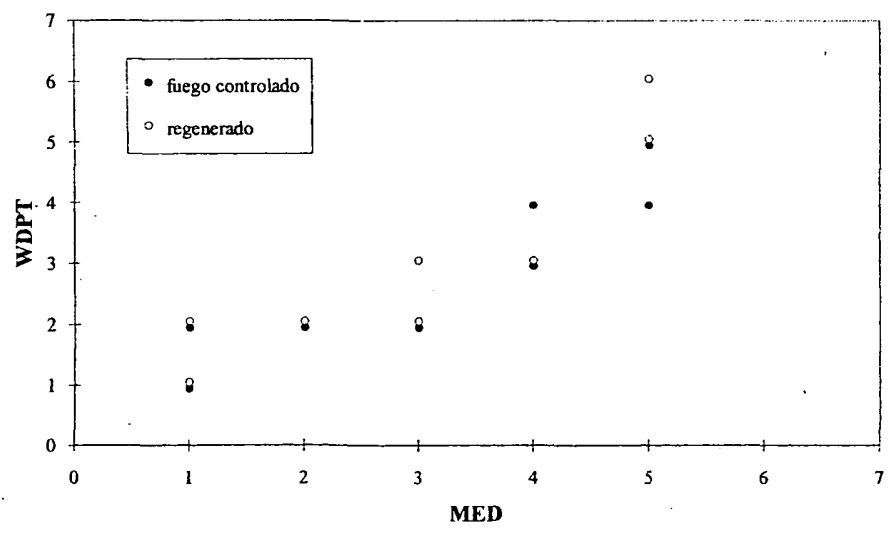

Figura 7. Comparación entre los resultados obtenidos con el MED y WDPT tests en ambas zonas de estudio.

Comparison between MED and WDPT tests results in both study sites.

\section{Discusión y conclusiones}

El uso de fuego controlado de baja intensidad, como medida de prevención y control de incendios forestales, es frecuente en algunas zonas montañosas de Portugal. Algunos estudios apuntan efectos beneficiosos de esta práctica sobre el ecosistema, ya que posibilita cambios en la composición de las comunidades vegetales, propicia la regeneración de especies así como el control de pestes o enfermedades, mejora la calidad de los pastos, etc. (REGO, 1986; BOTELHO, 1988). 
El análisis de algunas de las variables de los suelos estudiados demuestra que el fuego controlado, debido a su baja intensidad, no provoca ningún tipo de alteración en la densidad-porosidad, fracción gruesa, distribución textural, contenido de materia orgánica, ni tampoco en la humedad. Si bien el desfase cronológico entre el fuego controlado y el período de medición fue de 24 meses, MARTÍNEZ FERNÁNDEZ \& DÍAZ PEREIRA (1994) señalan también pocas diferencias apreciables en variables de tipo físico (densidad-porosidad y textura) y en el contenido de materia orgánica comparando una zona incendiada hacía 9 meses con una parcela testigo. Sin embargo, dichos autores sí encuentran importantes diferencias en las propiedades hídricas de los suelos, destacando, entre otras, una reducción de la humedad en la zona afectada por el fuego. En el caso estudiado, aunque se ha observado un mayor nivel de retención hídrica en los suelos regenerados, debido a su menor insolación y a la mayor capacidad de las plantas para fijar el agua en el ecosistema, las diferencias de humedad han sido poco notables, posiblemente por la reducción de la capacidad de infiltración en esta zona por la hidrofobia.

Los resultados demuestran una localización clara de la repelencia al agua en la parte de la cuenca no sometida al fuego controlado y ocupada de forma mayoritaria por Erica sp. Si bien WALLIS \& HORNE (1992) encuentran una relación significativa entre contenidos altos de materia orgánica en el suelo y comportamientos hidrófobos severos, en nuestro caso no podemos argumentar lo mismo, ya que los valores de materia orgánica son altos en la zona afectada por el fuego controlado y sin embargo la hidrofobia es irrelevante. BARRET \& SLAYMAKER (1989), BROCK \& de BANO (1990) indican que la repelencia al agua es independiente de la cantidad de materia orgánica. No obstante, de acuerdo con BISDOM et al. (1993), para la aparición de hidrofobia es esencial la presencia de compuestos orgánicos de origen vegetal (residuos de raíces, hojas y troncos) revistiendo las partículas del suelo y también formando parte de los componentes intersticiales del propio suelo. Sin embargo, no todas las plantas producen sustancias hidrófobas.

Aunque los suelos arenosos, con un contenido en arcillas inferior al $5 \%$, facilitan la adhesión de las sustancias hidrófobas a sus partículas, en suelos francos y con un buen grado de agregación, como los estudiados, también puede manifestarse la hidrofobia. En la cuenca de Valtorto predomina la agregación de tipo granular, detectada al tamizar las muestras, siendo este hecho muy importante debido a que la formación de agregados favorece que éstos puedan ser fácilmente cubiertos por las sustancias hidrófobas según ya indicaron Mc GUIE \& POSNER (1980). Esta circunstancia nos permite conectar con la relación establecida en diversos trabajos entre la humedad del suelo y la hidrofobia, ya que al aumentar el contenido de agua en el suelo durante las fases húmedas del año se produce un proceso de desagregación y la posterior 
desaparición de la hidrofobia según ya indicaron CROCKFORD et al. (1991) y puede comprobarse en la Figura 4.

Debido al contraste existente entre la uniformidad de las variables consideradas, salvo la cubierta vegetal en relación con el fuego controlado, y la localización espacial del comportamiento hidrófobo del suelo, sugerimos la hipótesis de asociar dicha hidrofobia a la presencia de Erica sp. GIOVANNINI \& LUCCHESI (1983) ya detectaron la aparición de hidrofobia en asociación con especies típicas del chaparral mediterráneo, entre ellas algunas ericáceas, en el estudio del efecto del fuego sobre los suelos en una parcela experimental en Cerdeña. Al igual que en el caso estudiado, el efecto del fuego hizo desaparecer las características hidrófobas previas al incendio y se requirió un período de tres años para volver a detectar hidrofobia en el lugar estudiado (GIOVANNINI et al., 1987).

Una de las consecuencias principales producto de una alteración brusca sobre un ecosistema determinado (en nuestro caso un fuego controlado) es la posible sustitución de las comunidades existentes por otras nuevas, que pueden evolucionar hacia la comunidad preexistente o no. Si bien la desaparición de la hidrofobia como consecuencia del fuego se atribuye a que altas temperaturas (por encima de los $280^{\circ} \mathrm{C}$ ) destruyen por combustión los elementos orgánicos que repelen el agua (de BANO et al., 1976), también puede argumentarse que fuegos de menor intensidad pueden provocar la desaparición de la hidrofobia al destruirse las fuentes de las sustancias hidrófobas, en nuestro caso haciendo desaparecer de determinados espacios las ericáceas. Si comparamos los gráficos 3 y 4 y la información de la tabla 5 se observa la relación existente entre la distribución de Erica sp. y la presencia en diverso grado de la hidrofobia: si en la mitad inferior del transecto de la zona regenerada se aprecia una relación entre el porte de dicho arbusto e intensidad de la hidrofobia, es significativo que la única muestra tomada en una zona carente de vegetación (punto correspondiente a los $85 \mathrm{~m}$ del transecto) tuviera un valor de hidrofobia muy bajo. Se han realizado de manera aleatoria una serie de muestreos en otros puntos pertenecientes a la misma unidad geográfica (Serra de Lousâ), detectándose valores de hidrofobia muy altos (clases 5 y 6 ) en algunas laderas donde los ejemplares de Erica sp. superan los 2,5 m de altura, en zonas no alteradas durante al menos 20 años.

En relación a los métodos utilizados para la medición de la hidrofobia, podemos afirmar que el grado de replicación encontrado, teniendo como margen una clase, es más bien discreto en comparación con el 99 \% que aparece en DOERR (1998) utilizando un número de muestras similar. Al respecto, podemos comentar que mientras en el campo se trabaja con una muestra inalterada y sobre la superficie del suelo, en el laboratorio se trabaja con una muestra totalmente alterada y correspondiente a los primeros $5 \mathrm{~cm}$ del suelo. 
Ello explica que el grado de hidrofobia de las muestras correspondientes al área de fuego controlado sea mayor en el laboratorio que en circunstancias naturales: la posibilidad de restos de residuos orgánicos productores de hidrofobia en el horizonte subsuperficial del suelo puede manifestarse en el laboratorio, pero no en el campo, al aplicar gotas sobre una muestra alterada y homogeneizada al ser tamizada.

Los resultados de laboratorio demuestran que, si bien a escala de ladera (zonas de fuego controlado y regenerada) y métrica (transectos) la variabilidad espacial de la hidrofobia es apreciable', a escala milimétrica existe una gran uniformidad ya que el $100 \%$ de las gotas (WDPT) utilizadas en el test se agrupan en dos clases siempre contiguas. También los datos de laboratorio indican un buen nivel de correspondencia y ajuste entre los resultados obtenidos con el MED y WDPT tests. Al contrario que las observaciones de DOERR (1998), que aprecia una ausencia de correspondencia en las clases 5 (suelo muy hidrófobo) del MED test, a la que corresponde un rango de clases en el WDPT que va desde la 3 (ligeramente hidrófobo) hasta la 7 (extremadamente hidrófobo), no se observa ninguna clase con un comportamiento disperso. Estudios como el de HARPER \& GILKES (1994), que han hallado un alto nivel de correspondencia entre ambos métodos, han llegado a expresar estar relación en una ecuación.

El principal interés de la hidrofobia en la investigación en curso radica en su relación con los procesos hidrológicos y erosivos. Los resultados preliminares (COELHO et al., 1998) demuestran que en sucesos de lluvia, independientemente de la intensidad de la misma, ocurridos sobre suelos secos en la cuen$\mathrm{ca}$, los coeficientes de escorrentía en las laderas regeneradas han sido mayores que en las afectadas por el fuego controlado, donde se ha detectado cierta discontinuidad espacial en el flujo superficial. La continuidad espacial de la escorrentía superficial en la zona hidrófoba está totalmente demostrada al detectarse la existencia de picos en la curva del hidrograma registrado en la salida de la cuenca. Ello además supone una pérdida de agua, durante los períodos secos del año, en la parte vegetada de la cuenca que puede interpretarse como un mecanismo de defensa de la vegetación nativa (Erica sp.) para evitar la competencia y colonización de otras especies según la idea expresada en CARTER et al. (1994). A pesar de una mayor escorrentía en la zona vegetada en relación con la hidrofobia, la pérdida de suelo en esta zona es muy pequeña debido a la interceptación de la energía cinética de las gotas de lluvia.

Durante el período de tiempo en el que los suelos de la cuenca han estado por encima del umbral de humedad delimitado para la aparición de la hidrofobia $(14 \%)$, la respuesta hidrológica ha sido inversa, siendo los coeficientes de escorrentía notablemente superiores en la zona sometida a fuego controlado y con mayor superficie de suelo desnudo, así como las tasas de erosión. 
De cara al futuro, la paulatina colonización del área afectada por el fuego controlado de especies vegetales preexistentes, como Erica sp., puede alterar el comportamiento hidrogeomorlógico de la cuenca. En este punto la mayor o menor intensidad del pastoreo de cabras, como elemento regulador de la vegetación, va a jugar un papel fundamental.

Agradecimientos. El presente proyecto de investigación está financiado por el Programa PAMAF del Ministerio de Agricultura y Pesca del Gobierno de Portugal. El primer autor se ha beneficiado de una beca postdoctoral de investigación financiada por la Consejería de Educación y Juventud de la Junta de Extremadura y el Fondo Social Europeo.

\section{Referencias}

ADAMS, S., STRAIN, B. R. \& ADAMS, M. S. (1969). Water-repellent soils and annual plant cover in a desert scrub community of South Eastern California. Proc. Symp. Water-repellent soils, Univ. of California, May 1968: 289-295.

de BANO, L. F. \& LETEY, J. (Eds.) (1969). Proceedings of the symposium on waterrepellents soils. University of California, Riverside, May 6-10, 1968.

de BANO, L. F., MANN, L. D. \& HAMILTON, D. A. (1970). Translocation of hydrophobic substances into soil by burning organic litter. Proceedings of the Soil Science Society of America, 34: 779-782.

de BANO, L. F., SAVAGE, S. M. \& HAMILTON, A. D. (1976). The transfer of heat and hydrophobic substances during burning. Proceedings of the Soil Science Society of America, 40: 779-782.

BARRETT, G. \& SLAYMAKER, O. (1989). Identification, characterisation and hydrological implications of water repellency in mountain soils, southern British Columbia. Catena, 16: 477-489.

BISDOM, E. B. A., DEKKER, L. W. \& SCHOUTE, J. F. T. (1993). Water repellent of sieve fractions from sandy soils and relationships with organic material and soil structure. Geoderma, 56: 105-118.

BOTELHO, H. S. (1988). Notas sobre o fogo controlado. Relatório de Provas de Aptidâo Pedagógica e Cientifica. UTAD, Vila Real.

BROCK, J. H. \& de BANO, L. F. (1990). Wettability of an Arizona chaparral soil influenced by prescribed burning. USDA Forestry Service General Technical Report, R.M.-191: 206-209.

BURCH, G. J., MOORE, I. D. \& BURNS, J. (1989). Soil hydrophobic effects on infiltration and catchment runoff. Hydrological Processes, 3: 211-222.

CARTER, D. J., HETHERINGTON, R. E., MORROW, G. \& NICHOLSON, D. (1994). Trends in water repellency measurements from soils sampled at 
ANÁLISIS DE LA REPELENCIA AL AGUA EN UNA PEQUEÑA CUENCA HIDROGRÁFICA...

different soil moisture and land use. Proceedings of the Second National Water Repellency Worshop, Perth (Western Australia): 49-57.

CERDÀ, A., SCHNABEL, S., CEBALLOS, A. \& GÓMEZ AMELIA, D. (1998). Soil hydrological response under simulated rainfall in the Dehesa Ecosystem, Extremadura, SW Spain. Earth Surface Processes and Landforms, 23: 195-209.

COELHO, C. O. A., FERREIRA, A. J. D., CEBALlOS, A., BOULET, A. K. \& CARVALHO, T. M. M. (1998). Hydrology and soil erosion in areas subject to prescribed burning and extensive grazing and browsing by goats. Experimental design and first results. Proceedings of International Seminar and Field Trip on Land Degradation and Desertification, International Geographical Union. Aveiro, Portugal: 125-129.

CROCKFORD, H., TOPADILIS, S. \& RICHARDSON, D. P. (1991). Water repellency in a dry sclerophyll eucalypt forest -measuments and processes-. Hydrological Processes, 5: 405-420.

DEKKER, L. W. (1998). Moisture variability resulting from water repellence in Dutch soils. Doctoral Thesis, Wageningen Agricultural University, The Netherlands, $240 \mathrm{pp}$.

DEKKER, L. W., RITSEMA, C. J., OOSTINDIE, K. \& BOERSMA, O. H. (1998). Effect of drying temperature on the severity of soil water repellency. Soil Science, 163-10: 780-796.

DOERR, S.H. (1997). Soil Hydrophobocity: a review. Aveiro-Swansea Erosión Research Bulletin $\mathrm{n}^{\circ}$ 5, $44 \mathrm{pp}$.

DOERR, S. H. (1998). On standardizing the "water drop penetration time» and the "molarity of an ethanol droplet» techniques to classify soil hydrophobicity: a case study using medium textured soils. Earth Surface Processes and Landforms, 23: 663-668.

DOERR, S. H., SHAKESBY, R. A. \& WALSH, R. P. D. (1996). Soil hydrophobicity variations with depth and particle size fraction in burned and unburned Eucalyptus globulus and Pinus pinaster forest terrain in the Águeda Basin, Portugal. Catena, 27: 25-47.

DOERR, S. H., SHAKESBY, R. A. \& WALSH, R. P. D. (1998). Spatial variability of soil hydrophobocity in fire-prone eucalyptus and pine forest, Portugal. Soil Science, 163(4): 313-324.

FERREIRA, A. J. D. (1996). Processos hidrológicos e hidroquímicos em povoamentos de Eucalyptus globulus Labill. e Pinus pinaster Aiton. Unpublished Ph. D. thesis, Departamento de Ambiente e Ordenamento, Universidade de Aveiro, Portugal.

FERREIRA, A. J. D., CEBAllos, A. \& COELHO, C. O. A. (1998). Comportamiento hidrófobo del suelo en el oeste peninsular. Metodología y primeros resultados. En GÓMEZ ORTIZ, A. \& SALVADOR FRANCH, F. 
(Eds.): Investigaciones recientes de la Geomorfología española: 521-528. Barcelona.

FRANCO, C. M. M., TATE, M. E., \& OADES, J. M. (1994). The development of water repellency in sands: studies on the physico-chemical and biological mechanisms. Proceedings of the Second National Water Repellency Workshop, Perth, Westerm Australia: 18-30.

GIOVANNINI, G. \& LUCCHESI, S. (1983). Effect of fire on hydrophobic and cementing substances of soil aggregates. Soil Science, 136 (4): 231-236.

GIOVANNINI, G., LUCCHESI, S. \& GIACHETTI, M. (1987). The natural evolution of a burned soil: a three-year investigation. Soil Science, 143(3): 220226.

HARPER, R. J. \& GILKES, R. J. (1994). Soil attributes related to water-repellency and the utility of soil survey for predicting its occurrence. Australian Journal of Soil Research, 32: 1109-1124.

HENDRICKX, J. M. H., DEKKER, L. W. \& BOERSMA, O. H. (1993). Unstable wetting fronts in water repellent field soils. Journal of Environmental Quality, 22: 109-118.

IMESON, A. C., VERSTRATEN, J. M., van MULLINGEN, E. J. \& SEVINK. J. (1992). The effects of fire and water repellency on infiltration and runoff under Mediterranean type forests. Catena 19: 345-361.

JUNGERIUS, P. D. \& de JONG. J. H. (1989). Variability of water repellency in the dunes along the Dutch coast. Catena 16: 491-497.

KING, P. M. (1981). Comparison of methods for measuring severity of water repellence of sandy soils and assessment of some factors that affect its measurement. Australian Journal of Soil Research, 19: 275-285.

LETEY, J. (1969). Measurement of contact angle, water drop penetration time, and critical surface tension. Proceedings of the Symposium on Water-Repellent Soils, 6-10 May 1968, University of California, Riverside: 43-47.

MARTÍNEZ FERNÁNDEZ, J. \& DÍAZ PEREIRA, E. (1994). Changes of the physical and chemical properties in a soil affected by forest fire in Sierra Larga (Murcia, Spain). En SALA, M. \& RUBIO, J. L. (Eds.) Soil erosion and degradation as a consequence of forest fires: 67-77. Geoforma Ediciones.

Mc GUIE, D. A. \& POSNER, A. M. (1980). Water repelence of a heavy-textured western Australia surface soil. Australian Journal of Soil Research, 27: 797-806.

MEEUWIG, R. O. (1971). Infiltration and water repellency in granitic soils. USDA Forest Service, Research Paper INT-111.

MOORE, G. \& BLACKWELL, P. (1998). Water repellence. In MOORE, G. (Eds): Soil Guide, a handbook for managing agricultural soils. Agriculture Westerm Australian Bulletin 4343: 53-63. 
ANÁLISIS DE LA REPELENCIA AL AGUA EN UNA PEQUEÑA CUENCA HIDROGRÁFICA...

REGO, F. C. (1986). Effects of prescribed fire on vegetation and soil properties in Pinus pinaster forest of Nothern Portugal. Unpublished Ph. D. Dissertation. University of Idaho (USA).

RITSEMA, C. J. (1998). Flow and transport in water repellent sandy soils. Doctoral Thesis. Wageningen Agricultural University, The Netherlands, 215 pp.

ROBERTS, F. J. \& CARBON, B. A. (1971). Water repellence in sandy soils of south-western Australia. 1. Some studies related to fiel occurrence. Division of Plant Industry CSIRO (Australia), Field Station Record, 10: 13-20.

SCOTT, D. F. (1991). The influence of eucalypts of soil wettability. En Schönau, A.G. (Ed.): Proceedings of ILIFRO Symposium. Intensive Forestry: the role of eucalypts. Vol. 2: 1044-1056.

SCOTT, D. F. (1993). The hydrological effects of fire in South African mountain catchments. Journal of Hydrology 150: 409-432.

SCOTT, D. F., VERSFELD, D. B. \& LESCH, W. (1998). Erosion and sediment yield in relation to afforestation and fire in the mountains of the Western Cape Province, South Africa. South African Geographical Journal 80-1: 52-59.

SEVINK, J., IMESON A. C. \& VERSTRATEN, J. M. (1989). Humus form development and hillslope runoff, and the effects of fire and management, under Mediterranean forest in NE Spain. Catena 16: 461-475.

SHAKESBY, R. A., COELHO, C. O. A., FERREIRA, A. J. D., TERRY J. P. \& WALSH. R. P. D. (1993). Wildfire impacts on soil erosion and hydrology in wet Mediterranean forest, Portugal. International Journal of Wildland Fire 3, 95-110.

WALLIS, M. G. \& HORNE, D. J. (1992). Soil water repellency. Advances in Soil Science, 20: 91-146.

WALSH, R. P. D., BOAKES, D., COELHO, C. O. A., GONÇALVES, A. J. B., SHAKESBY, R. A. \& THOMAS, A. D. (1994). Impact of fire-induced hydrophobocity and post-fire forest litter on overland flow in nothern and central Portugal. Proceedings of the Second International Conference on Forest Fire Research, Coimbra, Portugal, vol. II: 1149-1159.

WILKINSON, J. E. \& MILLER, R. H. (1978). Investigation and treatment of localized dry spots on sand golf greens. Agronomy Journal, 66: 299-304.

WILSON, G. V., JARDINE, P. M., LUXMOORE, R. J. \& JONES, R. J. (1990). Hydrology of a forested hillslope during storm events. Geoderma, 46: 119-138.

YORK, C. (1998). Water repellent soils as they occur on UK golf greens. International Workshop on Soil Water Repellency: origins, assesment, occurrence, consequences, modelling and amelioration. Wageningen, Netherlands, pp. 18. 